\title{
Modeling cross-language structural priming in sentence production
}

\author{
Yung Han Khoe (yhkhoe@protonmail.com) \\ CLS, Radboud University \\ Nijmegen, The Netherlands \\ Chara Tsoukala (x.tsoukala@gmail.com) \\ CLS, Radboud University \\ Nijmegen, The Netherlands \\ Gerrit Jan Kootstra (g.kootstra@let.ru.nl) \\ CLS, Radboud University \\ Nijmegen, The Netherlands \\ Stefan Frank (s.frank@let.ru.nl) \\ CLS, Radboud University \\ Nijmegen, The Netherlands
}

\begin{abstract}
A central question in the psycholinguistic study of multilingualism is how syntax is shared across languages. We implement a model to investigate whether error-based implicit learning can provide an account of cross-language structural priming. The model is based on the Dual-path model of sentence-production (Chang, 2002). We implement our model using the Bilingual version of Dual-path (Tsoukala, Frank, \& Broersma, 2017). We answer two main questions: (1) Can structural priming of active and passive constructions occur between English and Spanish in a bilingual version of the Dualpath model? (2) Does cross-language priming differ quantitatively from within-language priming in this model? Our results show that cross-language priming does occur in the model. This finding adds to the viability of implicit learning as an account of structural priming in general and cross-language structural priming specifically. Furthermore, we find that the within-language priming effect is somewhat stronger than the cross-language effect. In the context of mixed results from behavioral studies, we interpret the latter finding as an indication that the difference between cross-language and withinlanguage priming is small and difficult to detect statistically.
\end{abstract}

Keywords: cross-language structural priming; multilingualism; sentence production; syntax; dual-path model

\section{Introduction}

Psycholinguistic studies investigating syntax in both monolingual as well as multilingual speakers are often based on the structural priming paradigm. Structural priming is the tendency of speakers to reuse syntactic structures that they have previously encountered. In the study by J. K. Bock (1986) that introduced the paradigm, participants were more likely to use a passive target sentence (e.g., "The church is being struck by lightning") after repeating a passive sentence ("The referee was punched by one of the fans") than after repeating an active prime sentence ("One of the fans punched the referee"). Over 15 years ago, a number of studies showed that structural priming also occurs between two different languages (Hartsuiker, Pickering, \& Veltkamp, 2004; Loebell \& Bock, 2003; Meijer \& Fox Tree, 2003).

Several studies have found no difference between the strength of within-language and cross-language structural priming (Hartsuiker, Beerts, Loncke, Desmet, \& Bernolet, 2016; Kantola \& van Gompel, 2011; Schoonbaert, Hartsuiker, \& Pickering, 2007). In contrast, Cai, Pickering, Yan, and Branigan (2011) and Bernolet, Hartsuiker, and Pickering (2013) did find a stronger within-language than crosslanguage structural priming effect. This quantitative difference was accounted for by Bernolet et al. (2013) under the assumption that less proficient speakers of the second language (L2) had not yet developed syntactic representations that were shared across languages, or at least not for the syntactic structure under investigation. This would suggest that a prerequisite for equally strong within- and cross-language structural priming is that speakers are highly proficient in both languages.

Competing theoretical accounts of structural priming have been proposed. In the theoretical model introduced by Pickering and Branigan (1998), the residual activation of syntactic representations and combinatorial nodes leads to repeated use of particular syntactic representations. A bilingual version of this residual activation account was proposed by Hartsuiker et al. (2004). An alternative account explains structural priming as a form of error-based implicit learning (Chang, Dell, \& Bock, 2006). According to this account, error-based learning causes changes in the extent to which different syntactic structures are expected to occur. When a prime sentence is processed, the connections associated with its syntactic structure are strengthened, making that structure's occurrence more expected. This learning mechanism affects the production of the target sentence as it increases the likelihood of producing the same structure. In this account, structural priming is therefore regarded as a long-lasting effect. Support for this view comes from a large number of studies that have demonstrated that structural priming can last over time and persists over the processing of other sentences (K. Bock \& Griffin, 2000; Boyland \& Anderson, 1998; Branigan, Pickering, Stewart, \& McLean, 2000; Hartsuiker \& Kolk, 1998; Huttenlocher, Vasilyeva, \& Shimpi, 2004; Saffran \& Martin, 1997). 
Here, we investigate whether implicit learning can also account for cross-language priming. We do so by taking a wellknown monolingual model of structural priming, and extending it to the bilingual case.

\section{The Dual-path model}

Different implemented cognitive models of monolingual structural priming have been introduced by Chang (2002), Malhotra (2009), and Reitter, Keller, and Moore (2011). We use Chang's (2002) Dual-path model, that explains a wide range of sentence production phenomena in a number of different languages.

Dual-path is an implicit learning model of sentence production. It is a connectionist model which is based on the Simple Recurrent Network (SRN; Elman, 1990) architecture. The first pathway in the model is the sequencing system, that learns how words are ordered in a sentence, while the second pathway acquires meaning-to-word-form mappings. Dual-path has been used to investigate monolingual structural priming in English (Chang et al., 2006) and German (Chang, Baumann, Pappert, \& Fitz, 2015). Both of these studies demonstrated structural priming in the model, and thus provide support for the implicit learning account.

The model has also demonstrated the potential to account for experimental data from various second language acquisition and production studies. A Korean-English bilingual Dual-path model was used to examine the interaction between the effect of the age of acquisition and input factors, such as length of exposure, on second-language sentence production (Janciauskas \& Chang, 2018). A Spanish-English bilingual version of this model was recently developed to investigate cross-linguistic transfer (Tsoukala et al., 2017) and codeswitching (Tsoukala, Frank, van den Bosch, Valdéz Kroff, \& Broersma, 2019). So far, no studies have been reported that demonstrate cross-language structural priming in the model.

\section{The present study}

We perform a computational modeling experiment to further test the viability of implicit learning as an account of structural priming in general and cross-language structural priming specifically. We do this by ascertaining whether cross-language structural priming can occur in the Dual-path model. We simulate cross- and within-language priming of actives and passives, using artificial versions of Spanish and English. Furthermore, we investigate if cross-language priming differs quantitatively from within-language priming in the model.

We expect cross-language structural priming to occur, because cross-language structural priming has been experimentally demonstrated by Hartsuiker et al. (2004) in adults, and by Vasilyeva et al. (2010) in children, for the languages and syntactic structures used in the present work. Additionally, as mentioned above, within-language priming has been shown to occur in the model (Chang et al., 2006, 2015). Finally, a bilingual version of the Dual-path model has demonstrated the ability to code-switch, without being exposed to codeswitched language (Tsoukala et al., 2019) and code-switching has been interpreted as an indication that syntax is shared between languages (Kootstra, Van Hell, \& Dijkstra, 2010; Loebell \& Bock, 2003).

Assuming the model does display cross-language structural priming, we have no strong expectation of whether or not it will differ in strength from within-language priming. However, we aim to meet the suggested prerequisite for equivalent within- and cross-language priming effects (Bernolet et al., 2013) by simulating balanced bilingual speakers, who are equally proficient in both languages.

\section{Method}

\section{Model}

To simulate participants in a cross-language priming experiment, we trained the Bilingual Dual-path $\operatorname{model}^{1}$ (Figure 1) to simulate simultaneous Spanish-English bilinguals, who start acquiring both Spanish and English from infancy.

The Bilingual Dual-path model is a modified version of the original Dual-path model (Chang, 2002). The training input to the model consists of sentences in an artificial language that are paired with messages that encode their meaning (see examples below, under: Artifical languages). The model learns to convert a message into a sentence by predicting the sentence word by word. A difference between the Dual-path architecture and other Recurrent Neural Networks is that the network has connections with fixed weights between concepts and roles of the message to be expressed.

Artificial languages Both artificial languages ${ }^{2}$ we used include the same twelve sentence types: Animate intransitive, Animate with-intransitive, Inanimate intransitive, Locative, Transitive (in active or passive form), Cause-motion, Transfer dative (in prepositional object (PO) form), Benefactive dative (in PO form), Benefactive transitive, State-change, and Locative alternation $^{3}$. The two languages together have 275 unique lexical items. In addition to nouns, verbs, adjectives, determiners, and prepositions, these lexical items include inflectional morphemes such as a past tense marker (Spanish: '-pas'; English: '-pst') and a past participle marker (Spanish: '-prf'; English: '-par'). The message semantics contain 121 concepts, and 7 thematic roles. These numbers differ somewhat from those that were preregistered (see Section Pre-registered analysis). This is because we made small adjustments to the auxiliary verbs and inflectional morphemes of the artificial languages. None of these changes lead to different answers to our research questions. Only singular verbs, pronouns, nouns, and adjectives were used. Verbs and pro-

\footnotetext{
${ }^{1}$ The Bilingual Dual-path model can be downloaded from: https://github.com/xtsoukala/dual_path

${ }^{2}$ The files that the model requires to generate the artificial language input, and the input for the priming experiment can be found here: https://osf.io/pm6f9/ (2006)

${ }^{3}$ Examples for these sentence types can be found in Chang et al.
} 


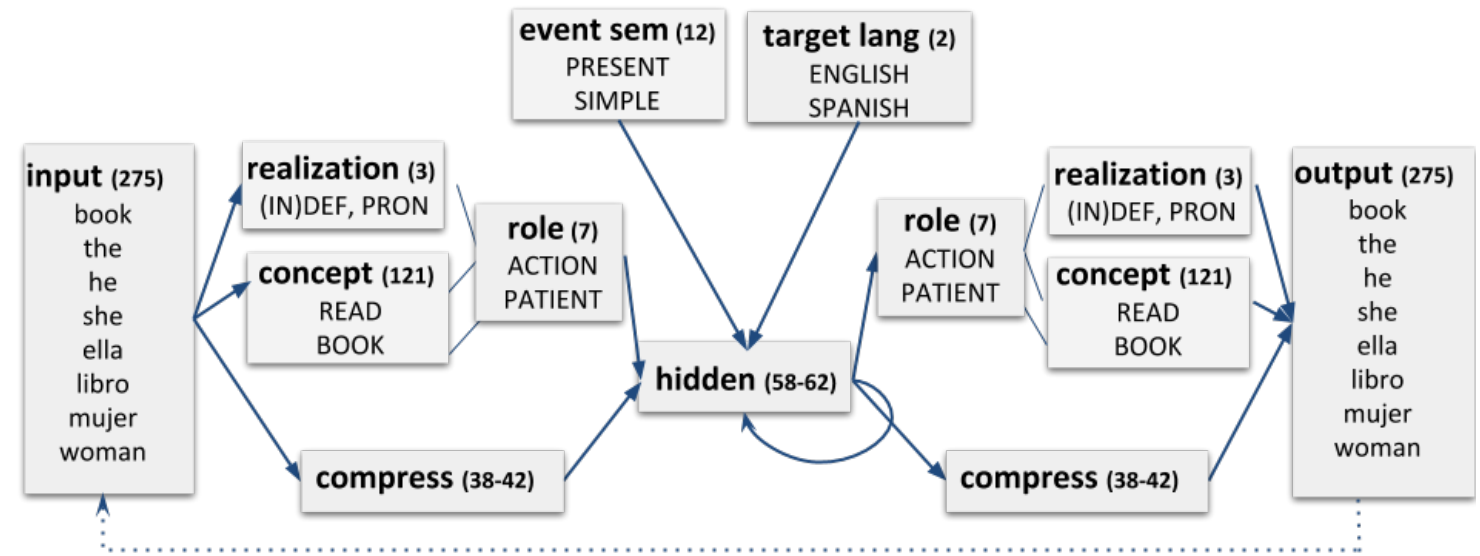

Figure 1: Bilingual Dual-path, the model used in our priming experiment. The model is a next-word prediction model that converts messages into sentences. It is an SRN-based model (the lower path, via the 'compress' layers) that is augmented with a semantic stream (upper path) that contains information about concepts, thematic roles, event semantics, and the target language. The number of units per layer are shown in parentheses. The numbers of units for the hidden and compress layers vary across simulations. (Figure adapted from Tsoukala et al. (2017))

nouns were always in third person form.

Because our aim is to verify the possibility of structural priming between different languages, we designed artificial versions of English and Spanish that maximize the likelihood of revealing an effect. If a structure is produced very frequently irrespective of priming, a small increase caused by priming might not result in a detectable effect. We addressed this issue by using balanced frequencies of the structures under investigation. This means that actives (see examples 1, 2 below) and passives (examples 3,4) occur with the same frequency in the training input we provide the model. For similar reasons, we model balanced bilingual speakers by training the model on both languages from the beginning and on almost equal numbers of sentences in the two languages, that randomly deviate only marginally.

In the training and test input, each message that can be expressed using two different syntactic structures has a strong bias towards one of those structures. This was done by creating differences in activation based on how each structure emphasizes thematic roles in the sentence. Biasing towards an active sentence $(1,2)$, for example, was done by giving the agent a higher activation $(\mathrm{X}: 1)$ than the patient $(\mathrm{Y}: 0.5$ or Y:0.75). In the same way, a bias towards a passive sentence $(3,4)$ was achieved with a higher activation for the patient (Y:1), than for the agent (X:0.5 or X:0.75). Similarly to Chang et al. (2006), we gave the target messages in the priming experiment a weaker bias than we used in the training and test input by giving the de-emphasized roles an activation of 0.95 instead of 0.5 or 0.75 .

1. Spanish Active: el padre romper -pas la botella .

$\mathrm{X}=$ def, FATHER, M;

ACTION-LINKING = BREAK;

$\mathrm{Y}=$ def, BOTTLE;

EVENT-SEM = X:1, Y:0.5, PAST,

SIMPLE, ACTION-LINKING;

TARGET-LANG $=$ es
2. English Active: the father break -pst the bottle .

$[\ldots]$;

EVENT-SEM = X:1, Y:0.5, PAST, SIMPLE, ACTION-LINKING; TARGET-LANG = en

3. Spanish Passive: la botella es romper -prf por el padre . $[\ldots]$;

EVENT-SEM = X:0.5, Y:1, PAST, SIMPLE, ACTION-LINKING; TARGET-LANG $=$ es

4. English Passive: the bottle is break -par by the father .

$[\ldots]$;

EVENT-SEM = X:0.5, Y:1, PAST, SIMPLE, ACTION-LINKING; TARGET-LANG $=$ en

Training and testing model accuracy A set of 8,000 unique message-sentence pairs was generated for each model participant. $80 \%$ of these sentences were used for training, while $20 \%$ were set aside for testing the accuracy of the model. Following Chang et al. (2006), the message was excluded from $25 \%$ of training pairs. The models iterated over their training sets 20 times. After each of these 20 epochs, model accuracy was tested using the test set. The training set was shuffled at the beginning of each epoch.

Model configuration The models have a number of hidden layer units that was sampled from a uniform distribution between 58 and 62, and a number of compress layer units sampled from a uniform distribution between 38 and 42. The fixed weight value for concept-role connections was sampled from a uniform distribution between 13 and 17 . The sentences are approximately equally divided over the two languages, where the language percentage of English was sampled from a uniform distribution between 48 to $52 \%$ and the rest was Spanish. Other than this, we used the model's default settings. 


\section{Priming experiment}

Model participants Some of the model participants we trained did not successfully learn the artificial languages. We therefore trained 120 models and selected the 80 model participants with the highest meaning accuracy (i.e. percentage of grammatically correct sentences that convey the target message without any additions, over all test sentences). The accuracy scores for these models varied from $70.5 \%$ to $87.9 \%$. A supplementary analysis includes all 120 model participants.

Experimental trials Independent of the training and test sets, a single set of experimental trials was generated that was used to perform the priming experiment on all of the model participants. Each trial consisted of a combination of a unique prime sentence and a unique target message that did not have any semantic overlap in terms of their verb, agent, and patient. With two types of LANGUAGE COMBINATION trials and two types of PRIME LANGUAGE trials, we had four possible combinations of prime- and target-language: English-English, Spanish-Spanish, Spanish-English, and English-Spanish. We had equal numbers of these four language combinations, which in turn means that there were equal numbers of withinand cross-language trials. We also had equal numbers of trials with active and passive primes, and equal numbers of trials with active- and passive-bias target-messages. The two types of trials for Prime Structure, LANGUAGe CombiNATion, Prime Language, and Target-Message Bias combine for a total of 16 different trial types. We had 50 prime-target combinations that all occurred as each of the 16 different trial types. This means that each experiment consisted of 800 trials.

Procedure The priming experiment was performed on the models after 20 training epochs. As was done in Chang et al. (2006) and Chang et al. (2015), we presented the models with prime sentences without a message, and with learning turned on in the model. After each prime, a response was elicited from the model by presenting it with a target message.

After each trial, the connection weights were reset to the values they had before starting the priming experiment. The state in which the model encounters each trial was thus the same for all of the trials, hence, there was no between-trial priming or any other learning effects during the experiment. This means that the order of the trials did not need to be (pseudo-)randomized across model participants.

For the priming experiment, the learning rate was set to 0.2 . In our pre-registration we reported that a learning rate of 1.2 would be used there, but this was an error in the preregistration. The exploratory experiment on within-language priming in fact used a learning rate of 0.2 during the priming experiment, and this was also the intended learning rate for the pre-registered experiment. This difference with the preregistration does not increase the probability of finding an effect. If anything, a higher learning rate would have resulted in a larger priming effect (Chang et al., 2006).

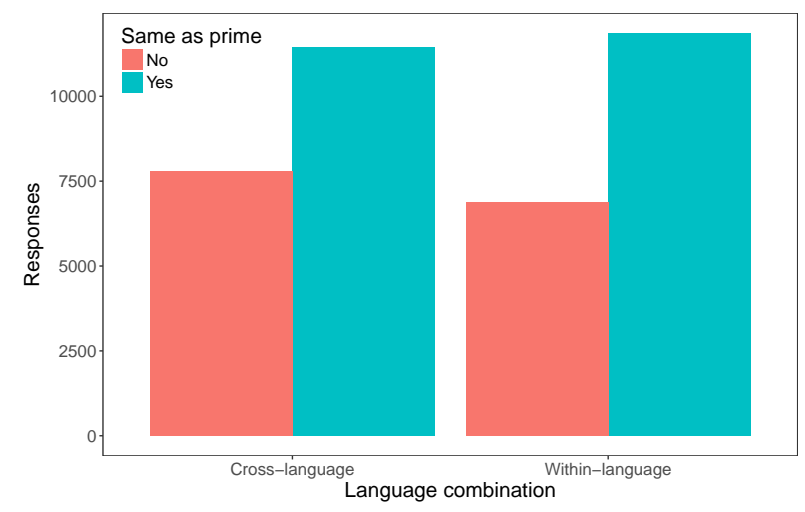

Figure 2: Total number of responses that had either the same structure as the prime or the alternative structure, split by within- or cross-language trials. The plot shows that there were more same structure than alternative structure responses for both LANGUAGE Combination conditions. However, the difference was larger for within-language trials.

\section{Results}

Our analysis only included those responses that correctly conveyed the target message, either with an active or a passive structure. However, we disregarded errors involving definiteness of articles or missing periods. This means we included $60 \%$ of the responses on cross-language trials, and $59 \%$ of responses on within-language trials. On cross-language trials, model participants produced 11,451 sentences $(59 \%$ of sentences) with the same structure as the prime, while producing 7,802 sentences $(41 \%)$ with the alternative structure. On within-language trials, 11,856 responses $(63 \%)$ had the same structure as the prime, whereas 6,877 responses (37\%) had the alternative structure. Figure 2 shows that there were more same-structure than alternative-structure responses for both LANGUAGE COMBINATION conditions, but that the difference was larger for within-language trials.

\section{Pre-registered analysis}

As pre-registered ${ }^{4}$, we analyzed the data from our experiment with a Bayesian logistic mixed-effects model, using a logit link function, using the function brm from the package brms (Bürkner et al., 2017; Bürkner, 2018, version 2.12.0) in $\mathrm{R}$ ( $\mathrm{R}$ Core Team, 2013, version 3.5.1). The model predicts a binary dependent variable, SAME AS PRIME, that indicates whether the sentence structure that the model produced and the structure of the prime sentence were the same (1), or different (0). In addition to the predictor of interest, LANGUAGE COMBINATION (Cross-language $=0$, Withinlanguage $=1)$, the model includes three contrast-coded covariates: Prime Structure (Active $=-0.5$, Passive $=0.5$ ), TARGET-MESSAGE BIAS (Active $=-0.5$, Passive $=0.5$ ), and Prime Language (English $=-0.5$, Spanish $=0.5$ ). We fit random intercepts for items and model participants, as well as by-item and by-participant random slopes for LANGUAGE COMBINATION. Regularizing priors were used in all our models, which give a minimal amount of information with

\footnotetext{
${ }^{4}$ The pre-registration can be accessed here: https:// aspredicted.org/728mn.pdf
} 
Table 1: Summary of the fixed effects in the Bayesian logistic mixed-effects model $(N=37,946)$. For each predictor are shown its estimate with $95 \%$ Bayesian credible interval and the posterior probability that the estimate is positive.

\begin{tabular}{lrrr}
\hline Predictor & Estimate & $95 \%$ CrI & $P($ Estimate $>0)$ \\
\hline INTERCEPT & 0.49 & {$[0.38,0.60]$} & 1.00 \\
LANGUAGE COMBINATION & 0.23 & {$[0.12,0.34]$} & 1.00 \\
PRIME LANGUAGE & 0.16 & {$[0.01,0.29]$} & 0.99 \\
PRIME STRUCTURE & 0.84 & {$[0.74,0.94]$} & 1.00 \\
TARGET-MESSAGE BIAS & -0.31 & {$[-0.41,-0.22]$} & 0.00 \\
LANGUAGE COMBINATION $\times$ PRIME LANGUAGE & -0.06 & {$[-0.25,0.13]$} & 0.27 \\
PRIME STRUCTURE $\times$ TARGET-MESSAGE BIAS & 2.98 & {$[2.79,3.19]$} & 1.00 \\
\hline
\end{tabular}

the objective of yielding stable inferences. Prior means were 0 , and did thus not bias towards specific effects. The standard deviations for the priors that we used for the predictors are based on the effect sizes that resulted from our preliminary analysis of within-language priming.

Unfortunately, even when using a large number of 16,000 iterations, 12 chains, and a high value of 0.99 for the adapt_delta parameter, this model did not result in valid and reliable parameter estimates. This was apparent from the large number of divergent transitions after warmup, and the low Bulk and Tail Effective Sample Sizes (ESS) (https://mc-stan.org/misc/warnings.html). Analysis of the model output revealed that the ESS values were specifically related to the estimates of the by-item random slopes for LANGUAGE COMBINATION. In addition, the credible interval (CrI) for these estimates were consistently close to zero across different numbers of iterations and chains, and different values for the adapt_delta parameter.

\section{Adjusted analysis}

Because of the reliability issues in the pre-registered regression model we removed the by-item random slopes and their correlations from the model, while leaving it unchanged otherwise. The resulting model yielded valid and reliable results, as evidenced by the absence of the type of warnings that the pre-registered model resulted in. Note, however, that both models revealed the same pattern of results that lead to the same answers to our research questions.

The regression analysis results are summarized in Table 1. The positive intercept, with a credible interval far from zero, shows a clear priming effect at the reference level (i.e., crosslanguage) of the LANGUAGE COMBINATION predictor. We interpret this as strong evidence for cross-language priming in the Dual-path model. The positive estimate for the LANGUAGE COMBINATION predictor, with a credible interval that does not cross zero, indicates that the within-language priming effect is stronger in the Dual-path model than the crosslanguage priming effect.

\section{Discussion}

The results of our experiment reveal a clear and strong crosslanguage structural priming effect. We thus provide evidence for the viability of implicit learning as an account of crosslanguage structural priming. In turn, our finding provides support for the implicit learning model implemented in Dualpath, as an account of structural priming in general. We should note, however, that this finding does not provide evidence against other implemented models of structural priming. The hybrid model introduced by Reitter et al. (2011), for example, also predicts cross-language structural priming. Fortunately, a way to empirically distinguish between this account and the Dual-path account is available. The former account predicts that priming will not occur between structures in different languages that do not have the same word order. The Dual-path account, on the other hand, does not seem to rule out such a priming effect.

We also find slightly stronger within- than cross-language structural priming. As a number of behavioral studies failed to find a significant difference between cross- and withinlanguage priming, this could suggest that our model provides an insufficiently adequate account of structural priming in this respect. However, other studies did find differences in the strength of cross-language and within-language priming, and these differences have been explained as resulting from participants' proficiency differences between the two languages. It might therefore be the case that we did not succeed in simulating sufficiently balanced bilinguals, although this is unlikely given the way our models were trained.

However, a simple account that could explain both the available behavioral results and our modeling results seems plausible. The presence of a small difference between crossand within-language priming, that is hard to detect statistically in highly proficient bilinguals, is consistent with the human data. This difference could become clearer if either proficiency differences increase, or if experimental methods are applied that are more likely to detect small effects. In addition, the absence of a significant effect is not proof that an effect does not exist (Vasishth \& Nicenboim, 2016).

In two of the four experiments reported on by Hartsuiker et al. (2016), for example, within-language priming was stronger than cross-language, even though this difference was not found to be significant. In the other two experiments, cross-language priming was either stronger than within-language priming or it depended on the prime lan- 
guage, but in any case none of the differences were statistically significant. In the first experiment conducted by Kantola and van Gompel (2011), within-language priming was nonsignificantly stronger than cross-language priming, whereas in the second experiment within-language priming was nonsignificantly weaker than cross-language priming. However, the difference in strength was larger in the first than in the second experiment. The results reported by Schoonbaert et al. (2007) and re-analysed by Hartsuiker et al. (2016); Hartsuiker and Bernolet (2017) showed non-significantly stronger within-language than cross-language priming when there was no semantic overlap between verbs in the prime and target sentences, as was the case in our comparison. When prime and target verbs were identical or translation-equivalent, however, Schoonbaert et al. (2007) found that within-language priming was significantly stronger than between-language priming. Overall, these results do not provide strong evidence that cross-language and within-language priming are equally strong.

Our analysis does reveal a clear effect of language combination. However, our experiment is relatively large in terms of the number of model participants and especially large in terms of the number of trials per participant. We performed a post-hoc analysis on a subset of our data that is more comparable to (but still larger than) the size of behavioral experiments. From the original 80 model participants, we excluded the 20 highest performing and the 20 lowest performing participants. We reduced the number of trials from 800 to 208 per participant while keeping the same distribution across conditions. The positive intercept resulting from this analysis still reveals a clear cross-language priming effect: Estimate $=0.38,95 \% \mathrm{CrI}=[0.19,0.57]$. In contrast, the LANGUAGE COMBINATION predictor now has a credible interval that crosses zero (Estimate $=0.19,95 \% \mathrm{CrI}=[-0.03$, $0.43]$ ). It therefore does not provide strong evidence anymore for a difference between cross- and within-language priming. If the model gives an approximately correct estimate of the difference between within- and cross-language priming, we cannot expect human studies to reveal that difference with the amount of data they have available. This is especially true if we consider that human data generally has a lower signalto-noise ratio than modeling data.

It could be costly to conduct an experiment with a large number of participants to verify that within-language priming is stronger than cross-language priming in balanced bilinguals. Likewise, increasing the number of experimental trials might cause concentration problems in participants. A way to address this might be to conduct the study as a large online experiment.

\section{Further work}

Relative proficiency in the two languages involved in crosslanguage structural priming can influence the strength of the priming effect. Now that we have established that crosslanguage priming occurs in the Dual-path model, a fruitful direction for future research will be to explore the relationship between second language proficiency and structural priming between languages.

As argued by, for example, Bernolet, Hartsuiker, and Pickering (2009), structural priming could be a phenomenon that takes place at different levels (e.g., information structure and syntactic structure), and syntactic alternations are different in the extent to which these levels play a role. To reach a deeper understanding of structural priming, it is therefore important to extend our modeling to further syntactic alternations, such as datives and genitives.

\section{References}

Bernolet, S., Hartsuiker, R. J., \& Pickering, M. J. (2009). Persistence of emphasis in language production: A crosslinguistic approach. Cognition, 112(2), 300-317.

Bernolet, S., Hartsuiker, R. J., \& Pickering, M. J. (2013). From language-specific to shared syntactic representations: The influence of second language proficiency on syntactic sharing in bilinguals. Cognition, 127(3), 287-306.

Bock, J. K. (1986). Syntactic persistence in language production. Cognitive Psychology, 18(3), 355-387.

Bock, K., \& Griffin, Z. M. (2000). The persistence of structural priming: Transient activation or implicit learning? Journal of Experimental Psychology: General, 129(2), 177.

Boyland, J. T., \& Anderson, J. R. (1998). Evidence that syntactic priming is long-lasting. In Proceedings of the Twentieth Annual Conference of the Cognitive Science Society (Vol. 1205).

Branigan, H. P., Pickering, M. J., Stewart, A. J., \& McLean, J. F. (2000). Syntactic priming in spoken production: Linguistic and temporal interference. Memory \& Cognition, 28(8), 1297-1302.

Bürkner, P.-C. (2018). Advanced Bayesian Multilevel Modeling with the R Package brms. The R Journal, 10(1), 395411.

Bürkner, P.-C., et al. (2017). brms: An R package for Bayesian multilevel models using Stan. Journal of Statistical Software, 80(1), 1-28.

Cai, Z. G., Pickering, M. J., Yan, H., \& Branigan, H. P. (2011). Lexical and syntactic representations in closely related languages: Evidence from Cantonese-Mandarin bilinguals. Journal of Memory and Language, 65(4), 431445.

Chang, F. (2002). Symbolically speaking: A connectionist model of sentence production. Cognitive Science, 26(5), 609-651.

Chang, F., Baumann, M., Pappert, S., \& Fitz, H. (2015). Do lemmas speak German? a verb position effect in German structural priming. Cognitive Science, 39(5), 1113-1130.

Chang, F., Dell, G. S., \& Bock, K. (2006). Becoming syntactic. Psychological Review, 113(2), 234.

Elman, J. L. (1990). Finding structure in time. Cognitive Science, 14(2), 179-211. 
Hartsuiker, R. J., Beerts, S., Loncke, M., Desmet, T., \& Bernolet, S. (2016). Cross-linguistic structural priming in multilinguals: Further evidence for shared syntax. Journal of Memory and Language, 90, 14-30.

Hartsuiker, R. J., \& Bernolet, S. (2017). The development of shared syntax in second language learning. Bilingualism, 20(2), 219.

Hartsuiker, R. J., \& Kolk, H. H. (1998). Syntactic persistence in dutch. Language and Speech, 41(2), 143-184.

Hartsuiker, R. J., Pickering, M. J., \& Veltkamp, E. (2004). Is syntax separate or shared between languages? crosslinguistic syntactic priming in Spanish-English bilinguals. Psychological Science, 15(6), 409-414.

Huttenlocher, J., Vasilyeva, M., \& Shimpi, P. (2004). Syntactic priming in young children. Journal of Memory and Language, 50(2), 182-195.

Janciauskas, M., \& Chang, F. (2018). Input and agedependent variation in second language learning: A connectionist account. Cognitive Science, 42, 519-554.

Kantola, L., \& van Gompel, R. P. (2011). Between-and within-language priming is the same: Evidence for shared bilingual syntactic representations. Memory \& Cognition, 39(2), 276-290.

Kootstra, G. J., Van Hell, J. G., \& Dijkstra, T. (2010). Syntactic alignment and shared word order in code-switched sentence production: Evidence from bilingual monologue and dialogue. Journal of Memory and Language, 63(2), 210-231.

Loebell, H., \& Bock, K. (2003). Structural priming across languages. Linguistics, 41(5; ISSU 387), 791-824.

Malhotra, G. (2009). Dynamics of structural priming. Unpublished doctoral dissertation, University of Edinburgh.

Meijer, P. J., \& Fox Tree, J. E. (2003). Building syntactic structures in speaking: A bilingual exploration. Experimental Psychology, 50(3), 184.

Pickering, M. J., \& Branigan, H. P. (1998). The representation of verbs: Evidence from syntactic priming in language production. Journal of Memory and Language, 39(4), 633651.

R Core Team. (2013). R: A language and environment for statistical computing.

Reitter, D., Keller, F., \& Moore, J. D. (2011). A computational cognitive model of syntactic priming. Cognitive Science, 35(4), 587-637.

Saffran, E. M., \& Martin, N. (1997). Effects of structural priming on sentence production in aphasics. Language and Cognitive Processes, 12(5-6), 877-882.

Schoonbaert, S., Hartsuiker, R. J., \& Pickering, M. J. (2007). The representation of lexical and syntactic information in bilinguals: Evidence from syntactic priming. Journal of Memory and Language, 56(2), 153-171.

Tsoukala, C., Frank, S., van den Bosch, A., Valdéz Kroff, J., \& Broersma, M. (2019). Simulating Spanish-English codeswitching: El modelo está generating code-switches. In CMCL 2019: Workshop on Cognitive Modeling and Com- putational Linguistics.

Tsoukala, C., Frank, S. L., \& Broersma, M. (2017). “he’s pregnant": Simulating the confusing case of gender pronoun errors in L2 English. In the 39th Annual Meeting of the Cognitive Science Society (CogSci 2017) (pp. 33923397).

Vasilyeva, M., Waterfall, H., Gámez, P. B., Gómez, L. E., Bowers, E., \& Shimpi, P. (2010). Cross-linguistic syntactic priming in bilingual children. Journal of Child Language, 37(5), 1047-1064.

Vasishth, S., \& Nicenboim, B. (2016). Statistical methods for linguistic research: Foundational ideas-Part I. Language and Linguistics Compass, 10(8), 349-369. 